\title{
Doxorubicin-HPMA Conjugate
}

National Cancer Institute

\section{Source}

National Cancer Institute. Doxorubicin-HPMA Conjugate. NCI Thesaurus. Code C1366.

A copolymer conjug ate of the antineoplastic anthracycline doxorubicin and the watersoluble polymer N-(2-hydroxypropyl) methacrylamide (HPMA). Doxorubicin, an intercalator and a topoisomerase II inhibitor, prevents DNA replication and ultimately inhibits protein synthesis. This agent also generates oxyg en free radicals, resulting in cytotoxic lipid peroxidation of cell membrane lipid. HPMA conjug ation enhances the permeability and retention of this agent within the tumor vasculature. Poorly cleared by the lymphatic system, this formulation undergoes increased cleavage by tumor cell lysosomal proteinases, resulting in increased, sustained intracellular concentrations of free doxorubicin. Compared to other doxorubicin-containing formulations, this formulation may exhibit an improved toxicity profile due to the lower concentrations of free doxorubicin to which non-malignant tissues are exposed. 